\title{
Laser damage in silicon: energy absorption, relaxation and transport
}

\author{
A. Rämer, ${ }^{1, \text { a) }}$ O. Osmani, ${ }^{2}$ and B. Rethfeld ${ }^{1}$ \\ 1) Department of Physics and Research Center OPTIMAS, University of Kaiserslautern, 67663 Kaiserslautern, \\ Germany \\ ${ }^{2)}$ Faculty of Physics, University of Duisburg-Essen and CeNIDE, 47048 Duisburg, \\ Germany
}

(Dated: 18 September 2018)

Silicon irradiated with an ultrashort $800 \mathrm{~nm}$-laser pulse is studied theoretically using a two temperature description that considers the transient free carrier density during and after irradiation. A Drude model is implemented to account for the highly transient optical parameters. We analyze the importance of considering these density-dependent parameters as well as the choice of the Drude collision frequency. In addition, degeneracy and transport effects are investigated. The importance of each of these processes for resulting calculated damage thresholds is studied. We report damage thresholds calculations that are in very good agreement with experimental results over a wide range of pulse durations.

PACS numbers: 05.70.Ln, 79.20.Ap, 44.10.+i, 72.10.Di

\section{INTRODUCTION}

The interaction of laser pulses with solid matter has been subject of both experimental ${ }^{1-9}$ and theoretical $^{10-17}$ research for many years. Especially ultrashort laser pulses, that cause minimal collateral damage to the surrounding material, are of high interest for applications in medical surgery, micromachining and nanostructuring. ${ }^{18-21}$

For the theoretical description of laser-excited solids, both the timescale and the kind of material have to be carefully considered. ${ }^{22}$ During and directly after the excitation, the electron system is out of equilibrium and no temperature is defined. Consequently, nonequilibrium descriptions like the Boltzmann equation ${ }^{12,23-28}$ or kinetic Monte Carlo simulations ${ }^{29-32}$ have to be applied.

On a timescale of about a hundred femtoseconds ${ }^{3,11,27}$ after excitation, the electrons again follow an equilibrium distribution. A temperature can be assigned to both the electrons and the lattice. However, those temperatures will differ because the laser energy is mainly absorbed by the electrons and subsequently transferred to the lattice. Equilibration between both subsystems happens on a picosecond timescale. For metals, the well known two temperature model ${ }^{10}$ is often applied on this timescale to describe both heat relaxation and transport within the electron and lattice subsystem.

In contrast to the excitation of metals, it is not sufficient to follow carrier and lattice temperatures when describing laser-excited semiconductors. Because the free carrier density in these materials can vary over several orders of magnitude during irradiation, its evolution has to be treated explicitly. In addition, the highly transient free carrier density causes huge changes in the optical properties of the solid. Thus, a theoretical description of laser excitation of semiconductors has to account for

\footnotetext{
a) Electronic mail: araemer@physik.uni-kl.de
}

the transient free carrier density, transient optical properties, carrier and lattice temperature evolution as well as energy and particle transport.

This paper extends an existing two temperature description for semiconductors first presented by van Driel $^{11}$ to account for the transient optical properties during the excitation with a femtosecond-laser pulse. To that end, we will first introduce the theoretical model and the extension. Afterwards, we will investigate the influence of the transient optical parameters as well as degeneracy and transport effects on the results of the simulations. We will discuss the importance of each property and process under investigation by calculating damage thresholds and comparing with experimental data.

\section{THE DENSITY-DEPENDENT TWO TEMPERATURE MODEL}

In laser-irradiated semiconductors, electrons are excited from the valence to the conduction band via single or multiphoton absorption. Thus, electron-hole pairs are created. The order of the absorption process depends on the photon energy in relation to the band gap energy of the material. In our case, the irradiation of silicon with $800 \mathrm{~nm}$-laser pulses, the photon energy is larger than the indirect band gap of silicon. Here, it is sufficient to consider single and two photon absorption processes. Higher order processes are less probable and can, thus, be neglected. ${ }^{33}$ In addition, free carriers (electrons in the conduction band and holes in the valence band) can absorb further photons via free carrier absorption thereby increasing their kinetic energy. Conduction band electrons with a sufficiently high kinetic energy may ionize additional valence band electrons via impact ionization. Furthermore, free electrons and holes may recombine via Auger recombination transferring the excess energy to another free electron or hole. Meanwhile, the carrier system can couple to the lattice system. The temperatures of both systems will equilibrate until a thermal equilib- 
rium state is reached.

All these processes as well as carrier and heat diffusion can be modeled with the density-dependent two temperature model (nTTM) first presented by van Driel ${ }^{11}$. For sake of completeness, we will in the following derive the original model following his work ${ }^{11}$ before expanding it to account for transient optical parameters during irradiation.

\section{A. Basic Assumptions}

In the framework of the nTTM, free electrons and holes are assumed to be thermalized into an equilibrium distribution function, usually a Fermi distribution

$$
f_{c}(\varepsilon)=\frac{1}{1+\exp \left[\frac{ \pm\left(\varepsilon-\mu_{c}\right)}{k_{B} T_{e}}\right]},
$$

at all times. The distribution functions of electrons and holes are assumed to posses different chemical potentials $\mu_{c}$, where $c$ stands for "carrier" and may be substituted by $e$ for electrons and $h$ for holes, but a common temperature $T_{e}$. Here, $k_{B}$ denotes the Boltzmann constant. The plus sign in the exponent in Eq. (1) associates with the electrons, while the minus sign associates with the holes. The assumption of thermalized carriers is, however, questionable at times shortly after laser-excitation. As thermalization will take on the order of $100 \mathrm{fs},{ }^{3,11,27}$ carrier temperature and distribution functions as well as chemical potentials may be interpreted as quasi-temperature, quasi-Fermi distribution functions and quasi-chemical potentials.

Conduction and valence band are treated as parabolic bands. Consequently, the densities of states (DOS) of electrons

$$
\begin{aligned}
D_{e}(\varepsilon) & =\frac{m_{e, \mathrm{DOS}}^{* 3 / 2}}{\hbar^{3} \pi^{2}} \sqrt{2\left(\varepsilon-\varepsilon_{C}\right)} \\
\text { and holes } \quad D_{h}(\varepsilon) & =\frac{m_{h, \mathrm{DOS}}^{* 3 / 2}}{\hbar^{3} \pi^{2}} \sqrt{2\left(\varepsilon_{V}-\varepsilon\right)}
\end{aligned}
$$

are applied, where $\varepsilon_{C}$ and $\varepsilon_{V}$ denote the lower edge of the conduction band and the upper edge of the valence band, respectively. A different constant DOS effective mass $m_{c, \text { DOS }}^{*}$ is assumed for electrons and holes, respectively, see Tab. I.

Figure 1 shows a sketch of the DOS of electrons and holes as well as their quasi-Fermi distributions and chemical potentials.

By integrating over the carrier distribution multiplied with the respective DOS, the local carrier density reads

$$
n_{c}=2\left(\frac{m_{c, \mathrm{DOS}}^{*} k_{B} T_{e}}{2 \pi \hbar^{2}}\right)^{3 / 2} \mathcal{F}_{1 / 2}\left(\eta_{c}\right) .
$$

Here, the function

$$
\mathcal{F}_{\xi}\left(\eta_{c}\right)=\frac{1}{\Gamma(\xi+1)} \int_{0}^{\infty} \frac{x^{\xi}}{1+\exp \left(x-\eta_{c}\right)} \mathrm{d} x
$$

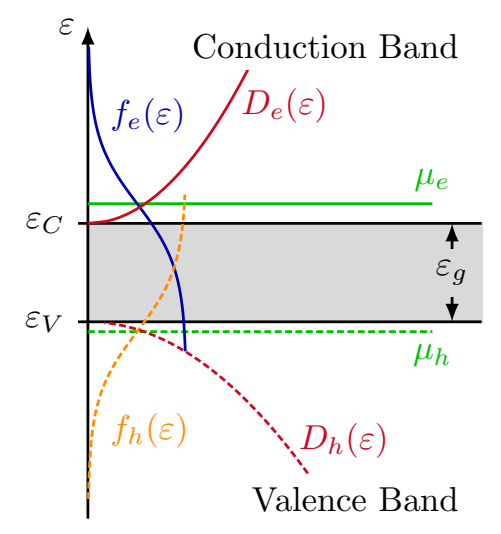

FIG. 1. (Color online) Sketch of DOS, quasi-Fermi distribution and quasi-chemical potentials of electrons and holes.

denotes the Fermi integral of order $\xi$. The so-called reduced Fermi levels of electrons and holes are defined as

$$
\eta_{e}=\frac{\mu_{e}-\varepsilon_{C}}{k_{B} T_{e}} \quad \text { and } \quad \eta_{h}=\frac{\varepsilon_{V}-\mu_{h}}{k_{B} T_{e}},
$$

respectively. ${ }^{11}$ They indicate the position of the respective chemical potentials in relation to the band edges. If the reduced Fermi levels become positive, the respective chemical potentials are positioned inside the respective bands and the carrier system is degenerate.

\section{B. Carrier and Energy Current}

In a laser-generated free carrier system, electrons and holes basically move together as electron-hole pairs since the Dember field that builds up due to charge separation prohibits carrier and current densities from becoming significantly different (ambipolar diffusion). ${ }^{11}$ We can therefore assume, that

$$
n_{e}=n_{h} \quad \text { and } \quad \vec{j}_{e}=-\vec{j}_{h}
$$

at each point in space. Under these conditions, the carrier current density is given by

$$
\begin{aligned}
\vec{J}= & -D\left\{\vec{\nabla} n_{e}+\frac{n_{e}}{k_{B} T_{e}}\left[H_{-1 / 2}^{1 / 2}\left(\eta_{e}\right)+H_{-1 / 2}^{1 / 2}\left(\eta_{h}\right)\right]^{-1} \vec{\nabla} \varepsilon_{g}\right. \\
& \left.+\frac{n_{e}}{T_{e}}\left[2 \frac{H_{0}^{1}\left(\eta_{e}\right)+H_{0}^{1}\left(\eta_{h}\right)}{H_{-1 / 2}^{1 / 2}\left(\eta_{e}\right)+H_{-1 / 2}^{1 / 2}\left(\eta_{h}\right)}-\frac{3}{2}\right] \vec{\nabla} T_{e}\right\},
\end{aligned}
$$

where $\varepsilon_{g}=\varepsilon_{C}-\varepsilon_{V}$ denotes the band gap energy, $H_{\zeta}^{\xi}\left(\eta_{c}\right)=\mathcal{F}_{\xi}\left(\eta_{c}\right) / \mathcal{F}_{\zeta}\left(\eta_{c}\right)$ is the ratio of Fermi integrals (4) and

$$
\begin{aligned}
D= & \frac{k_{B} T_{e}}{e} \frac{\mu_{e}^{0} \mu_{h}^{0} H_{1 / 2}^{0}\left(\eta_{e}\right) H_{1 / 2}^{0}\left(\eta_{h}\right)}{\mu_{e}^{0} H_{1 / 2}^{0}\left(\eta_{e}\right)+\mu_{h}^{0} H_{1 / 2}^{0}\left(\eta_{h}\right)} \\
& \times\left[H_{-1 / 2}^{1 / 2}\left(\eta_{e}\right)+H_{-1 / 2}^{1 / 2}\left(\eta_{h}\right)\right]
\end{aligned}
$$


with the carrier mobility $\mu_{c}^{0}$ is the ambipolar diffusion coefficient. Note that the band gap gradient $\vec{\nabla} \varepsilon_{g}$ is nonzero because of band gap shrinkage due to increased lattice temperature and carrier density during and after excitation. ${ }^{6,34,35}$

The heat current density $\vec{W}=\vec{w}_{e}+\vec{w}_{h}$ is the sum of electron and hole heat current densities and reads

$$
\begin{aligned}
\vec{W}= & \left\{\varepsilon_{g}+2 k_{B} T_{e}\left[H_{0}^{1}\left(\eta_{e}\right)+H_{0}^{1}\left(\eta_{h}\right)\right]\right\} \vec{J} \\
& -\left(\kappa_{e}+\kappa_{h}\right) \vec{\nabla} T_{e} .
\end{aligned}
$$

The expressions for the current densities include treatment of the Peltier and Seebeck effect as well as Ohm's and Fourier's law. A more detailed description and background information on the transport terms is given by van Driel. ${ }^{11}$

Note that whenever space charge effects cannot be neglected, for instance when studying Coulomb explosion, the assumption of ambipolar diffusion (6) is not valid anymore. Transport in this case has been studied by Bulgakova et al. ${ }^{14,15}$

\section{Energy and Particle Balance}

For the particle balance equation, interband laser absorption processes, namely single and two photon absorption, impact ionization, Auger recombination and carrier transport are considered:

$$
\frac{\partial n_{e}}{\partial t}=\frac{\alpha_{\mathrm{SPA}} I}{\hbar \omega_{L}}+\frac{\beta_{\mathrm{TPA}} I^{2}}{2 \hbar \omega_{L}}+\delta n_{e}-\gamma n_{e}^{3}-\vec{\nabla} \cdot \vec{J} .
$$

The first two terms describe single and two photon absorption, the third and fourth term stand for impact ionization and Auger recombination and the last term describes carrier transport. Here, $I$ denotes the intensity of the laser pulse, $\hbar \omega_{L}$ the photon energy, $\alpha_{\mathrm{SPA}}$ and $\beta_{\mathrm{TPA}}$ the single and two photon absorption coefficients, respectively, $\delta$ the coefficient for impact ionization and $\gamma$ the one for Auger recombination. All coefficients used in this work are given in Tab. I.

The total carrier energy density is given by

$$
\begin{aligned}
U_{e-h} & =\int_{-\infty}^{\varepsilon_{V}} \varepsilon D_{h}(\varepsilon) f_{h}(\varepsilon) \mathrm{d} \varepsilon+\int_{\varepsilon_{C}}^{\infty} \varepsilon D_{e}(\varepsilon) f_{e}(\varepsilon) \mathrm{d} \varepsilon \\
& =n_{e} \varepsilon_{g}+\frac{3}{2} n_{e} k_{B} T_{e}\left[H_{1 / 2}^{3 / 2}\left(\eta_{e}\right)+H_{1 / 2}^{3 / 2}\left(\eta_{h}\right)\right] .
\end{aligned}
$$

The first term in the second line represents the potential energy of the carrier pairs, while the second term gives the kinetic energy of electrons and holes.

The total carrier energy is modified by laser absorption, transport and carrier-phonon coupling. Thus, the balance equation for carrier energy density is

$$
\begin{aligned}
\frac{\partial U_{e-h}}{\partial t}= & \left(\alpha_{\mathrm{SPA}}+\alpha_{\mathrm{FCA}}\right) I+\beta_{\mathrm{TPA}} I^{2} \\
& -\vec{\nabla} \cdot \vec{W}-g\left(T_{e}-T_{p h}\right),
\end{aligned}
$$

where $\alpha_{\mathrm{FCA}}$ denotes the free carrier absorption coefficient. The carrier-phonon coupling parameter $g$ is nonconstant in semiconductors and highly depends on carrier density. It is therefore often described by $g=$ $C_{e-h} / \tau_{\mathrm{c}-\mathrm{ph}}^{\text {relax }}$, where the carrier heat capacity $C_{e-h}$ depends on the carrier density and $\tau_{\mathrm{c}-\mathrm{ph}}^{\mathrm{relax}}$ is the carrierphonon energy-relaxation time. ${ }^{11,36,37}$ This expression results from an analytical solution of the traditional TTM for metal films at times after laser-excitation, neglecting transport and assuming a constant heat capacity as well as a constant electron-phonon coupling parameter. Obviously, it is therefore questionable for semiconductors excited by ultrafast laser pulses. Comparison with carrier-phonon coupling parameters extracted with the Boltzmann equation nevertheless shows, that the approximation is justified whenever the carrier system is not highly degenerate. ${ }^{38}$

The lattice system is not directly heated up by the laser pulse but only indirectly via carrier-phonon coupling. Thus, the balance equation for lattice energy density reads

$$
\frac{\partial U_{p h}}{\partial t}=\vec{\nabla} \cdot\left(\kappa_{p h} \vec{\nabla} T_{p h}\right)+g\left(T_{e}-T_{p h}\right),
$$

where the first summand describes energy transport in the lattice system via Fourier's law and $\kappa_{p h}$ is the lattice thermal conductivity. The second term describes the energy exchange with the carrier system. This equation is basically identical to the one in the traditional TTM used for metals. ${ }^{10}$

To calculate carrier and lattice temperature, we have to look at the temporal derivatives of carrier and lattice energy densities in more detail. Lattice energy density only depends on lattice temperature. Thus, the temporal derivative of the lattice energy density can be expressed as

$$
\frac{\partial U_{p h}}{\partial t}=C_{p h} \frac{\partial T_{p h}}{\partial t},
$$

using the lattice heat capacity $C_{p h}=\partial U_{p h} / \partial T_{p h}$. For the carrier system things are more complicated because the carrier energy density does not only depend on carrier temperature but also on carrier density and band gap energy:

$$
\frac{\partial U_{e-h}}{\partial t}=C_{e-h} \frac{\partial T_{e}}{\partial t}+\frac{\partial U_{e-h}}{\partial n_{e}} \frac{\partial n_{e}}{\partial t}+\frac{\partial U_{e-h}}{\partial \varepsilon_{g}} \frac{\partial \varepsilon_{g}}{\partial t} .
$$

Note that the temporal derivative of band gap energy is non-zero, because the band gap depends on both carrier density and lattice temperature. ${ }^{6,11,34}$ Furthermore, to calculate the derivatives of carrier energy density, it is necessary to calculate the derivative of the reduced Fermi levels, as can be seen in Eq. (11). This is possible by taking the derivative with respect to carrier temperature, lattice temperature and carrier density, respectively, on both sides of Eq. (3), utilizing that all three are independent properties and solving for the derivative of the 
reduced Fermi level. The reduced Fermi level itself can be calculated by solving Eq. (3) for the Fermi integral and comparing with tabulated data that can, for instance, be generated using the GNU Scientific Library. ${ }^{39}$

\section{Non-Degenerate Carrier System}

If the chemical potentials of electrons and holes are located far outside the conduction and the valence band, respectively, only the Boltzmann tail of the Fermi distribution reaches inside the band. The carrier system is therefore non-degenerate and follows a Maxwell-Boltzmann distribution. Consequently, the reduced Fermi levels are large and negative and Fermi integrals of any order can be reduced to $\exp \left(\eta_{e}\right)$ and $\exp \left(\eta_{h}\right)$, respectively. The quotients of Fermi integrals $H_{\zeta}^{\xi}\left(\eta_{c}\right)$ tend towards one and the carrier energy and pair current densities simplify from Eqs. (7) and (9) to

$$
\vec{J}=-D\left(\vec{\nabla} n_{e}+\frac{n_{e}}{2 k_{B} T_{e}} \vec{\nabla} \varepsilon_{g}+\frac{n_{e}}{2 T_{e}} \vec{\nabla} T_{e}\right)
$$

with the ambipolar diffusivity $D=\frac{2 k_{B} T_{e}}{e} \frac{\mu_{\mu}^{0} \mu_{h}^{0}}{\mu_{e}^{0}+\mu_{h}^{0}}$ and

$$
\vec{W}=\left(\varepsilon_{g}+4 k_{B} T_{e}\right) \vec{J}-\left(\kappa_{e}+\kappa_{h}\right) \vec{\nabla} T_{e} .
$$

The carrier energy density in a non-degenerate system is given by

$$
U_{e-h}=n_{e} \varepsilon_{g}+3 n_{e} k_{B} T_{e},
$$

which immensely simplifies the calculation of the carrier heat capacity and other derivatives in Eq. (15).

Aside from the reduced Fermi levels being negative, another criterion for whether the carrier system is degenerate lies within the comparison of the carrier temperature $T_{e}$ with the Fermi temperature

$$
T_{F}=\frac{\hbar^{2}}{2 m_{c, \mathrm{DOS}}^{*} k_{B}}\left(3 \pi^{2} n_{e}\right)^{2 / 3},
$$

which is different for electrons and holes because of the different effective masses. Whenever the Fermi temperature is comparable to or higher than the carrier temperature, the respective carrier system is degenerate.

\section{E. Laser-Excitation}

To obtain the intensity of the laser pulse within the material, the attenuation in the direction of propagation is calculated with the one-dimensional ordinary differential equation (ODE)

$$
\frac{\mathrm{d} I}{\mathrm{~d} z}=-\left(\alpha_{\mathrm{SPA}}+\alpha_{\mathrm{FCA}}\right) I-\beta_{\mathrm{TPA}} I^{2},
$$

that includes linear interband or single photon absorption (SPA), linear intraband or free carrier absorption (FCA) and two photon interband absorption (TPA). Because of momentum conservation and the fact, that silicon is an indirect semiconductor, single photon processes can only happen under assistance of a phonon. Thus, the linear absorption coefficients $\alpha_{\mathrm{SPA}}$ and $\alpha_{\mathrm{FCA}}$ depend on lattice temperature. In addition, the FCA coefficient depends on carrier density because the more free carriers, the stronger the absorption. Because of the possibly steep carrier density and lattice temperature profiles within the material during irradiation, we have to account for spatial non-constant absorption coefficients when solving the ODE (20). Consequently, there is no closed-form analytical solution and the ODE (20) has to be solved numerically along with the balance equations (10), (12) and (13). The intensity transmitted through the material surface is

$$
I_{0}=\sqrt{\frac{4 \ln (2)}{\pi}} \frac{(1-R) \Phi}{\tau_{p}} \exp \left\{-4 \ln (2)\left[\frac{\left(t-t_{0}\right)}{\tau_{p}}\right]^{2}\right\},
$$

where $R$ denotes the reflectivity, $\Phi$ the fluence and $\tau_{p}$ the duration of the laser pulse that is centered around $t_{0}=3 \tau_{p}$.

Because of the usually large spot size of the laser pulse, the radial intensity distribution can be neglected and it is sufficient to describe absorption and transport in the direction of propagation at the center of the focus.

As already indicated above, the huge changes in free carrier density during the irradiation lead to significant changes in the optical parameters, namely the reflectivity and the FCA coefficient. Nevertheless, when modeling semiconductors, the reflectivity is often assumed to depend solely on lattice temperature. ${ }^{11,36,37,40}$ In these cases the FCA coefficient is written as a solely lattice temperature dependent cross-section multiplied by carrier density. In the following, we will denote these expressions for the reflectivity and the FCA coefficient as $T$-expression.

To account for the influence of the transient carrier density on reflectivity and FCA coefficient, we apply a Drude model. Considering the separate contributions of both, electrons and holes, the complex dielectric function in the framework of this model is given by ${ }^{41,42}$

$$
\begin{aligned}
\epsilon\left(\omega_{L}\right) & =\epsilon_{r}\left(\omega_{L}\right)-\frac{n_{e} e^{2}}{\epsilon_{0} \omega_{L}^{2}} \\
& \times\left[\frac{1}{m_{e, \text { cond. }}^{*}\left(1+i \frac{\nu_{e}}{\omega_{L}}\right)}+\frac{1}{m_{h, \text { cond. }}^{*}\left(1+i \frac{\nu_{h}}{\omega_{L}}\right)}\right] .
\end{aligned}
$$

Here, $\epsilon_{r}$ denotes the intrinsic dielectric constant, $m_{c, \text { cond. }}^{*}$ is the conductivity effective mass defined to reproduce electrical conductivity and susceptibility. ${ }^{43}$ The Drude collision frequency of electrons and holes, respectively, is denoted $\nu_{c}$. When assuming the collision frequencies of electrons and holes to be equal, $\nu_{\text {Drude }}=\nu_{e}=\nu_{h}$, 
and introducing a joint optical effective mass, $1 / m_{\mathrm{opt}}^{*}=$ $1 / m_{e, \text { cond. }}^{*}+1 / m_{h, \text { cond. }}^{*}$ Eq. (22) reduces to the usual Drude expression for the dielectric function. ${ }^{6,42}$

The complex refractive index $\tilde{n}=\sqrt{\epsilon}$, the reflectivity under normal incidence and the FCA coefficient

$$
R=\frac{|\tilde{n}-1|^{2}}{|\tilde{n}+1|^{2}} \quad \text { and } \quad \alpha_{\mathrm{FCA}}=\frac{2 \mathfrak{I m}(\tilde{n}) \omega_{L}}{c_{0}}
$$

can subsequently be calculated using Eq. (22). ${ }^{44}$ Here, $c_{0}$ denotes the speed of light in vacuum. While the steep carrier density profile within the medium during excitation may cause feedback effects and thus influence the reflectivity of the silicon sample ${ }^{6}$, these effects are estimated as negligible for all situations modeled here.

Concerning the collision frequency $\nu_{c}$, there is disagreement in literature on the question which kinds of collisions contribute (for an overview see review by Balling and $\mathrm{Schou}^{45}$ and references therein). Many authors assume a constant frequency $\nu_{\text {Drude }},{ }^{6,46-51}$ while others consider non-constant electron-electron and electronphonon collision frequencies. ${ }^{16,52-54}$

Electron-electron as well as hole-hole collisions, however, cannot contribute to the collision frequency entering the Drude model as both particles have the same effective mass and the collision does conserve the total carrier momentum. ${ }^{1,55,56}$ Electron-hole collisions, on the other hand, can contribute to the Drude collision frequency because of the different effective mass of both collision partners.

Sernelius ${ }^{57}$ as well as Hullin et al. ${ }^{55}$ investigated the importance of electron-hole collisions in comparison to carrier-phonon collisions and found that they can dominate under certain conditions. In the framework of the nTTM presented here, we thus consider both, electronhole and carrier-phonon collisions. We assume the electron-phonon and the hole-phonon collision frequency to be identical and proportional to lattice temperature: ${ }^{2}$

$$
\nu_{\mathrm{c}-\mathrm{ph}}=A T_{p h} .
$$

To our knowledge, there is no data for silicon or comparable semiconductors. For metals like gold, silver, copper or aluminum, Christensen et al. ${ }^{58}$ state that the proportionality constant $A$ is on the order of $1 \times 10^{11} \mathrm{~s}^{-1} \mathrm{~K}^{-1}$ to $4 \times 10^{11} \mathrm{~s}^{-1} \mathrm{~K}^{-1}$. Here, we assume that the proportionality constant for silicon is comparable to those for these metals and take $A=3.9 \times 10^{11} \mathrm{~s}^{-1} \mathrm{~K}^{-1}$ given for aluminum in Ref. 58.

The electron-hole collision frequency is calculated using

$$
\nu_{\mathrm{e}-\mathrm{h}}=\frac{\sqrt{\left\langle(\Delta v)^{2}\right\rangle}}{\langle\ell\rangle}
$$

where $\langle\ell\rangle$ denotes the mean free path of electrons and holes for screened Coulomb collisions and $\left\langle(\Delta v)^{2}\right\rangle=$ $2\left\langle U_{e}^{\mathrm{kin}}\right\rangle / m_{e}^{*}+2\left\langle U_{h}^{\mathrm{kin}}\right\rangle / m_{h}^{*}$ is the mean relative velocity squared of electrons and holes. The mean electron and hole kinetic energies $\left\langle U_{c}^{\text {kin }}\right\rangle$ can be calculated by splitting the second summand in Eq. (11), that represents the carrier kinetic energy, into the different parts for electrons and holes and dividing by carrier density. The mean free path of electrons and holes in a screened Coulomb potential is given by ${ }^{59}\langle\ell\rangle=\varkappa^{2} / n_{e} \pi$, where the inverse screening length $\varkappa$ for a free carrier gas can be calculated following Refs. 27, 60, and 61:

$$
\begin{aligned}
\varkappa^{2}= & \frac{e^{2}}{\epsilon_{0}}\left[\int_{\varepsilon_{C}}^{\infty} \frac{\partial D_{e}(\varepsilon)}{\partial \varepsilon} f_{e}(\varepsilon) \mathrm{d} \varepsilon+\int_{-\infty}^{\varepsilon_{V}} \frac{\partial D_{h}(\varepsilon)}{\partial \varepsilon} f_{h}(\varepsilon) \mathrm{d} \varepsilon\right] \\
= & \frac{e^{2}}{\epsilon_{0}} \frac{1}{\pi^{3 / 2} \hbar^{3}} \sqrt{\frac{k_{B} T_{e}}{2}} \\
& \times\left[\mathcal{F}_{-1 / 2}\left(\eta_{e}\right) m_{e, \mathrm{DOS}}^{* 3 / 2}+\mathcal{F}_{-1 / 2}\left(\eta_{h}\right) m_{h, \mathrm{DOS}}^{* 3 / 2}\right] .
\end{aligned}
$$

Note that this inverse screening length includes screening by both types of carriers, electrons and holes. Together with Eq. (25) this leads to an electron-hole collision frequency of

$$
\begin{aligned}
\nu_{\mathrm{e}-\mathrm{h}}= & \frac{\sqrt{3} \epsilon_{0} \pi\left(k_{B} T_{e}\right)^{3 / 2}}{e^{2}}\left[\frac{H_{1 / 2}^{3 / 2}\left(\eta_{e}\right)}{m_{e, \mathrm{DOS}}^{*}}+\frac{H_{1 / 2}^{3 / 2}\left(\eta_{h}\right)}{m_{h, \mathrm{DOS}}^{*}}\right]^{1 / 2} \\
& \times\left[H_{1 / 2}^{-1 / 2}\left(\eta_{e}\right)+H_{1 / 2}^{-1 / 2}\left(\eta_{h}\right)\right]^{-1}
\end{aligned}
$$

For a non-degenerate carrier system, this collision frequency reduces to

$$
\nu_{\mathrm{e}-\mathrm{h}}=\frac{\sqrt{3} \epsilon_{0} \pi\left(k_{B} T_{e}\right)^{3 / 2}}{2 e^{2}}\left[\frac{1}{m_{e, \mathrm{DOS}}^{*}}+\frac{1}{m_{h, \mathrm{DOS}}^{*}}\right]^{1 / 2} .
$$

The total electron and hole collision frequencies can now be calculated from the respective single contributions (24) and (27) using Matthiessen's rule. Note that, following (24) and (27), the total electron and the total hole collision frequency are identical.

\section{RESULTS}

The balance equations (10), (12) and (13) are solved in one dimension using a finite differences scheme. While an explicit forward time centered space (FTCS) scheme is used to obtain lattice temperature and carrier density, the carrier temperature is calculated using an iterative Crank Nicolson scheme. ${ }^{62}$ The equations are solved on a staggered grid, where the grid points for carrier and energy currents are positioned in between the grid points for temperatures and density. As initial conditions, we choose an equilibrium at $T_{e}(z, 0)=T_{p h}(z, 0)=300 \mathrm{~K}$ and, consequently, $n_{e}(z, 0)=10^{12} \mathrm{~cm}^{-3}$. As energyconserving boundary conditions, we use $J_{z}(z, t)=0$, $W_{z}(z, t)=0$ and $\kappa_{p h} \partial T_{p h} / \partial z=0$ at the surface $(z=0)$ and at $z=10 \mu \mathrm{m}$ assumed as the thickness of the material. The model parameters used for the calculations 


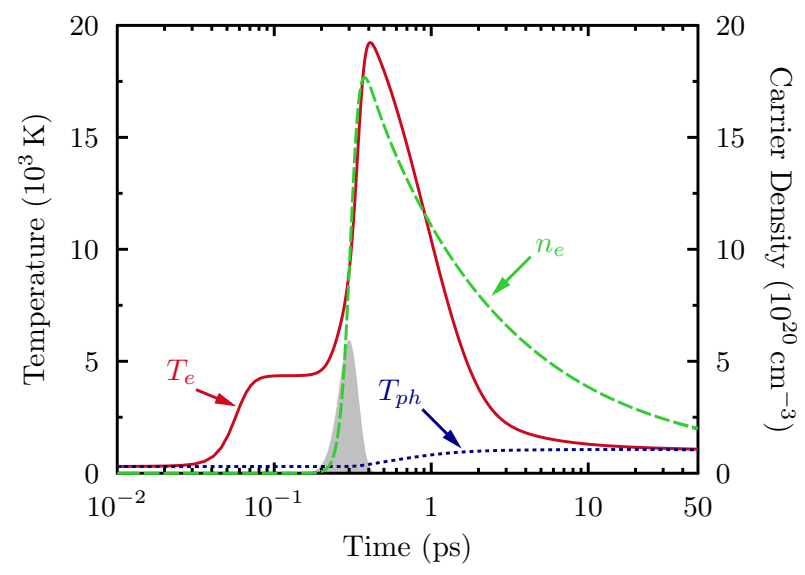

FIG. 2. (Color online) Characteristic temperature and density evolution at the surface of laser-irradiated silicon obtained with the nTTM. The laser intensity is sketched in arbitrary units as a gray area.

presented below are listed in Table I. It should be noted here that the nTTM like the commonly used TTM requires the use of various material parameters as well as approximations. While some of these parameters are affected with certain inaccuracies, numerous studies have proven the usefulness of the TTM in the past. ${ }^{58,63-66} \mathrm{Ob}-$ viously, it is possible to choose material parameters in such a way that the simulation gives the best agreement with the experiment, e.g. by fitting certain material parameters. However, we would like to point out, that no fitting was performed in the present simulations. The material parameters used here (cf. Tab. I) are all commonly accepted for the case of silicon. The sole exception is the carrier-phonon collision frequency (24). No value for the proportionality constant $A$ is know for silicon. The choice to use the value for aluminum from Ref. 58 is arbitrary and should not be understood as a fit.

For all figures showing temporal evolutions, a $100 \mathrm{fs-}$ laser pulse with a fluence of $130 \mathrm{~mJ} / \mathrm{cm}^{2}$ at $800 \mathrm{~nm}$ is applied.

Figure 2 shows the temporal evolution of carrier density (green dashed curve) and temperature (red solid curve) as well as lattice temperature (blue dotted curve) at the surface of laser-irradiated silicon. The data presented in the figure was calculated using the full model considering the transient optical parameters obtained with the Drude model, a Fermi distributed carrier system and transport.

The carrier temperature starts to increase early at very low intensities until it reaches a plateau of approximately $4360 \mathrm{~K}$ at about $90 \mathrm{fs}$. The first increase is due to linear absorption processes, to be specific SPA and to some extend FCA. The excess energy gained by these processes, namely all the energy that is not invested in overcoming the band gap, corresponds to a quasi-temperature of approximately $4380 \mathrm{~K}$. Due to carrier-phonon coupling the actual carrier temperature is slightly lower.
In the following $100 \mathrm{fs}$, carrier temperature only increases slightly before it ascends rapidly when the laser pulse approaches its maximum. At this point, on the one hand, TPA becomes important for the high intensities near the maximum of the pulse and, on the other hand, FCA becomes the dominant linear absorption process because of an increasing free carrier density.

The carrier temperature reaches its maximum slightly after the maximum of the laser pulse and then decreases mainly because of lattice heating.

Due to carrier-phonon coupling, carrier and lattice temperatures tend to equilibrate. Consequently, the lattice heats up at later times than the carriers. Total equilibration is not achieved for several tens of picoseconds because, even on this timescale, the carrier system is still heated up slightly as a result of Auger recombination.

The carrier density reaches its maximum shortly after the laser pulse and slightly earlier than carrier temperature. The maximum carrier density of $1.77 \times 10^{21} \mathrm{~cm}^{-3}$ is nine orders of magnitude larger than the initial carrier density. The increase is mainly due to photon absorption, while impact ionization appears to be insignificant. After reaching its maximum, the free carrier density starts to decrease due to Auger recombination.

In the following subsections, we will investigate the influence of several processes and properties on the outcome of our simulations. To further check the importance of the process or property under investigation and at the same time validate our model against the experiment, we present and discuss calculated damage thresholds at the end of each subsection. As a criterion for damage, we choose that the maximum lattice temperature reaches melting temperature $T_{\text {melt }}=1685 \mathrm{~K}$ as this represents the lowest fluence, at which damage can possibly occur. The damage threshold curve for the full model as well as experimental data published by Allenspacher et al. ${ }^{8}$ and Pronko et al. ${ }^{72}$ is always shown as a reference.

\section{A. Influence of Density-Dependent Optical Parameters}

In the last section, we demonstrated that the free carrier density in silicon may increase by as much as nine orders of magnitude during the irradiation with an ultrashort laser pulse. In the following, we will investigate the importance of the transient, density-dependent optical parameters. For simplicity, we assume here that the carrier system is non-degenerate and neglect carrier and energy transport.

Figure 3(a) depicts the reflectivity during the irradiation of silicon calculated using the $T$-expression (solid curve) and the Drude model (dashed curve), respectively, to determine both the reflectivity and the FCA coefficient as denoted in Tab. I. We immediately notice major differences: While the reflectivity calculated with the $T$-expression increases mirroring the behavior of lattice temperature, the reflectivity calculated with the Drude model decreases mirroring the inverse behavior of car- 
TABLE I. Model parameters for silicon.

\begin{tabular}{|c|c|c|c|}
\hline Quantity & Symbol & Value & Reference \\
\hline \multicolumn{4}{|c|}{ Band structure } \\
\hline Indirect band gap & $\varepsilon_{g}$ & $\left(1.16-\frac{7.02 \times 10^{-4} \mathrm{~K}^{-1} T_{p h}^{2}}{T_{p h}+1108 \mathrm{~K}}-1.5 \times 10^{-8} \mathrm{~cm} n_{e}^{1 / 3}\right) \mathrm{eV}$ & Refs. 6,11 , and 34 \\
\hline Electron DOS effective mass & $m_{e, \mathrm{DOS}}^{*}$ & $0.33 m_{e}$ & Ref. 36 \\
\hline Hole DOS effective mass & $m_{h, \mathrm{DOS}}^{*}$ & $0.81 m_{e}$ & Ref. 67 \\
\hline
\end{tabular}

Thermal and electrical properties

$\begin{array}{lclr}\text { Electron conductivity effective mass } & m_{e, \text { cond. }}^{*} & 0.26 m_{e} & \text { Ref. } 11 \\ \text { Hole conductivity effective mass } & m_{h, \text { cond. }}^{*} & 0.37 m_{e} & \text { Ref. } 11 \\ \text { Lattice heat capacity } & C_{p h} & \left(1.978+3.54 \times 10^{-4} \mathrm{~K}^{-1} T_{p h}-3.68 \mathrm{~K}^{2} T_{p h}^{-2}\right) \mathrm{J} /\left(\mathrm{cm}^{3} \mathrm{~K}\right) & \text { Refs. } 11 \text { and } 68 \\ \text { Lattice thermal conductivity } & \kappa_{p h} & 1585 \mathrm{~K}^{1.23} T_{p h}^{-1.23} \mathrm{~W} /(\mathrm{cm} \mathrm{K}) & \text { Refs. } 11 \text { and } 68 \\ \text { Melting temperature } & T_{\text {melt }} & 1685 \mathrm{~K} & \text { Ref. } 6 \\ \text { Carrier thermal conductivity } & \kappa_{\mathrm{e}-\mathrm{h}} & \left(-3.47 \times 10^{16}+4.45 \times 10^{14} \mathrm{~K}^{-1} T_{e}\right) \mathrm{eV} /(\mathrm{s} \mathrm{cm} \mathrm{K)} & \text { Refs. } 36 \text { and } 69 \\ \text { Carrier ambipolar diffusivity } & D & 18\left(300 \mathrm{~K} / T_{p h}\right) \mathrm{cm}^{2} / \mathrm{s} & \text { Ref. } 11 \\ \text { Auger recombination coefficient } & \gamma & 3.8 \times 10^{-31} \mathrm{~cm}^{6} / \mathrm{s} & \text { Refs. } 11 \text { and } 70 \\ \text { Impact ionization coefficient } & \delta & 3.6 \times 10^{10} \mathrm{exp}\left[-1.5 \varepsilon_{g} /\left(k_{B} T_{e}\right)\right] \mathrm{s}^{-1} & \text { Ref. } 11 \\ \text { Carrier-phonon relaxation time } & \tau_{\mathrm{c}-\mathrm{ph}}^{\text {relax }} & 0.5 \mathrm{ps} & \text { Ref. } 11\end{array}$

Single photon absorption coefficient

Two photon absorption coefficient

Free carrier absorption coefficient Reflectivity

Free carrier absorption coefficient

Reflectivity

Intrinsic dielectric constant
Optical properties at $800 \mathrm{~nm}$

$\begin{array}{lll}\alpha_{\mathrm{SPA}} & 1.12 \times 10^{3} \exp \left(T_{p h} / 430 \mathrm{~K}\right) \mathrm{cm}^{-1} & \text { Ref. } 40\end{array}$

$\beta_{\mathrm{TPA}} \quad 9 \mathrm{~cm} / \mathrm{GW}$

Ref. 40

Ref. 40

Ref. 40

Drude model

$\alpha_{\mathrm{FCA}} \quad$ calculated using Eq. (23)

Ref. 44

$R \quad$ calculated using Eq. (23)

Ref. 44

$\epsilon_{r} \quad 13.634+0.048 i$

Ref. 71 rier density. The reflectivity calculated with the Drude model already shows significant changes during irradiation, while the increase calculated with the $T$-expression is delayed and less pronounced.

Figure 3(b) clearly shows that the drastically different behavior of reflectivity has an impact on carrier and lattice temperatures as well as carrier density. The maxima of carrier density as well as carrier and lattice temperatures are all higher when using the Drude model than when using the $T$-expression. This is most prominent for carrier temperature but also noticeable for the final lattice temperature. The densities calculated using the Drude model and the $T$-expression, respectively, deviate at their maximum but tend towards similar values for later times. The reason is that Auger recombination, as a three particle process, is much stronger for higher densities.

The significant differences, especially in temperature, shown in Fig. 3(b) indicate that the choice of using either the $T$-expression or the Drude model to calculate reflectivity and FCA coefficient might strongly influence the damage thresholds estimated applying the nTTM.

In Fig. 4 the damage thresholds calculated using the $T$-expression (blue triangles) and the full model including density-dependent optical parameters (red diamonds) are shown in comparison to experimental data published by Allenspacher et al. ${ }^{8}$ and Pronko et al. ${ }^{72}$ as well as theoretical predictions presented by Chen et al. ${ }^{36}$. Both our calculations consider transport and a Fermi distributed carrier gas. For their calculations, Chen et al. ${ }^{36}$ solved an nTTM in combination with the $T$-expression. As damage criterion, they used a critical density fitted to match the experimental determined threshold for a pulse duration of $500 \mathrm{fs}$.

Figure 4 clearly shows, that our calculation using the $T$-expression significantly overestimates the damage thresholds while the damage thresholds calculated using the Drude model are in very good agreement with the 


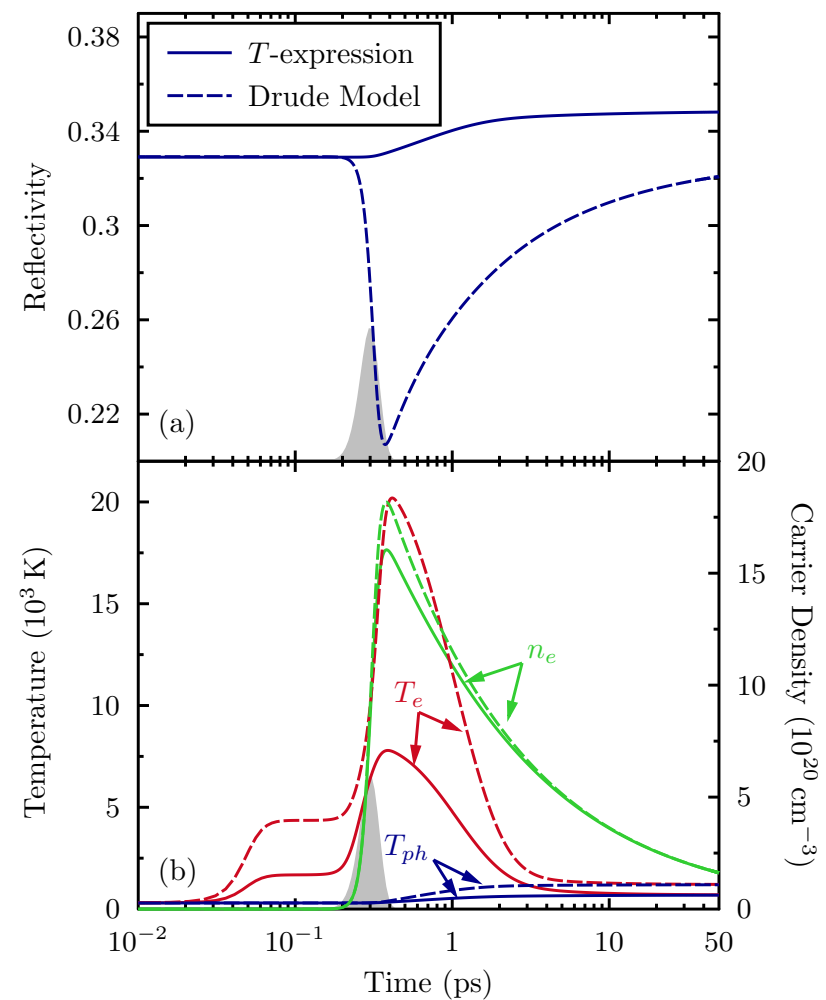

FIG. 3. (Color online) Reflectivity (a), carrier density as well as carrier and lattice temperature (b) calculated using either the $T$-expression or the Drude model.

experimental data even over a wide range of pulse durations from $80 \mathrm{fs}$ up to $6 \mathrm{ps}$. The thresholds calculated by Chen et al. ${ }^{36}$ agree reasonably well with the experiment. However, the threshold decreases for long pulses, whereas the experimental data does not show any decrease. For these pulse durations in the picosecond range, the thresholds calculated with our full model considering densitydependent optical parameters much better resemble the behavior of the experimental data.

We therefore conclude, that it is highly important to account for the transient optical parameters changing due to the strongly non-constant free carrier density in laserexcited silicon.

\section{B. Influence of Collision Frequency}

Following this conclusion, we will now look at the reflectivity obtained with the Drude model, especially the importance of the choice of carrier collision frequency, in more detail. We will again neglect transport and assume the carrier system to be non-degenerate at all times.

Figure 5(a) depicts the reflectivity calculated with the Drude model for different assumptions on the collision frequency. The reflectivity considering electron-hole and carrier-phonon collisions (red solid curve) is the same as

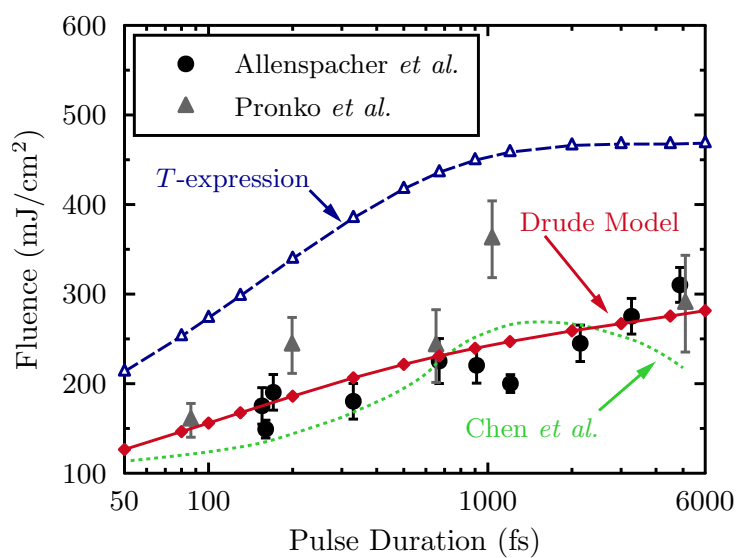

FIG. 4. (Color online) Damage thresholds calculated using either the T-expression or the full model and thereby the Drude model in comparison to experimental data obtained by Allenspacher et al. ${ }^{8}$ and Pronko et al. ${ }^{72}$ as well as thresholds simulated by Chen et al. ${ }^{36}$

shown in Fig. 3(a). In this case, the total electron and hole collision frequency varies from $118 \mathrm{THz}$ to maximum $500 \mathrm{THz}$. While the carrier-phonon collisions dominate the total collision frequency in the beginning for low intensities and later after the pulse, the electron-hole collisions dominate near the maximum of the laser pulse.

To investigate, whether the contribution of any type of collision to the Drude collision frequency can be neglected or it is even sufficient to assume a constant collision frequency, Fig. 5(a) additionally shows the reflectivity considering only electron-hole (green dash-dotted curve) or carrier-phonon collisions (blue dashed curve) as well as the reflectivity for a constant collision frequency of $^{6} 1 \mathrm{PHz}$ (orange dotted curve).

In all cases, the reflectivity follows the inverse behavior of the carrier density (gray dash-dot-dotted curve). The depths of the minima, however, are different. The reflectivity curves considering only electron-hole or carrierphonon collisions are nearly identical and show the deepest minimum, while the reflectivity for a constant collision frequency shows a less pronounced minimum.

The second optical property, that is directly influenced by the choice of the collision frequency, is the FCA coefficient. Figure 5(b) shows that all FCA coefficients roughly follow the behavior of carrier density. It is, however, obvious that the strength of FCA differs depending on the choice of the collision frequency. For the carrier density range considered here $\left(n_{e} \leq 7 \times 10^{21} \mathrm{~cm}^{-3}\right)$, the FCA coefficient predicted by the Drude model is higher for a higher collision frequency. This is directly reflected in the figure: the constant collision frequency of $1 \mathrm{PHz}$ is higher than the collision frequency considering both types of collisions at all times, as is the FCA coefficient. When only electron-hole or carrier-phonon collisions are considered, the collision frequency is always lower than when both are considered which is directly reflected in 


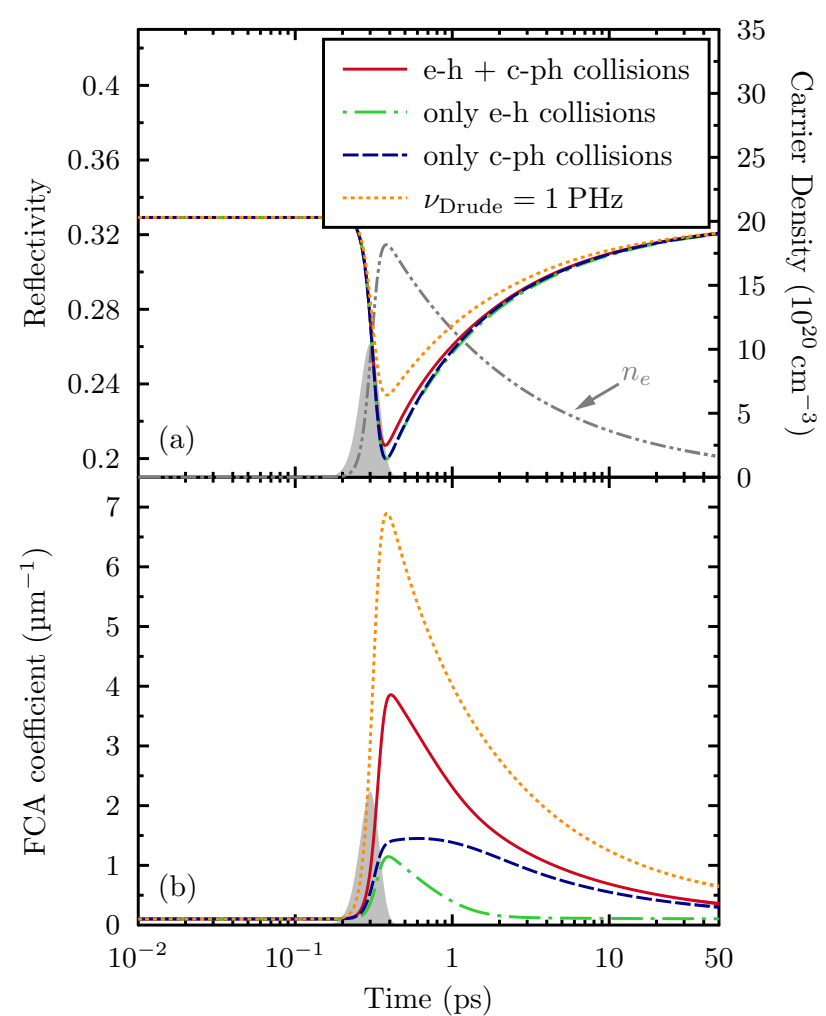

FIG. 5. (Color online) Reflectivity (a) and FCA coefficient (b) assuming a constant frequency of $1 \mathrm{PHz}$, considering either electron-hole collisions or carrier-phonon collisions or considering both, electron-hole and carrier-phonon collisions. As an orientation, the carrier density considering both types of collisions is shown as a gray dash-dot-dotted line. Note that the carrier density is nearly identical for all carrier collision frequencies shown here.

the corresponding FCA coefficients.

Comparing the FCA coefficients considering only one type of collision, we see that while the one for electronhole collisions decreases rapidly after the end of the laser pulse, the one for carrier-phonon collisions decreases much slower. This is because electron-hole collisions are mainly important at the maximum of the pulse, when the carrier temperature is highest, while carrier-phonon collisions stay important once the lattice temperature increases due to carrier-phonon coupling.

To further investigate the importance of the choice of the collision frequency, Fig. 6 shows carrier and phonon temperature for the different collision frequencies. Note that the carrier density is not shown here, because it is nearly identical in all cases. This can, however, not be said for the temperatures. The strongly different FCA coefficients shown in Fig. 5(b) lead to strongly different surface temperatures. While the highest lattice temperature, obtained for the constant frequency, is already above melting temperature, the lowest final temperatures, when only one type of collision is considered are between $840 \mathrm{~K}$ and $920 \mathrm{~K}$.

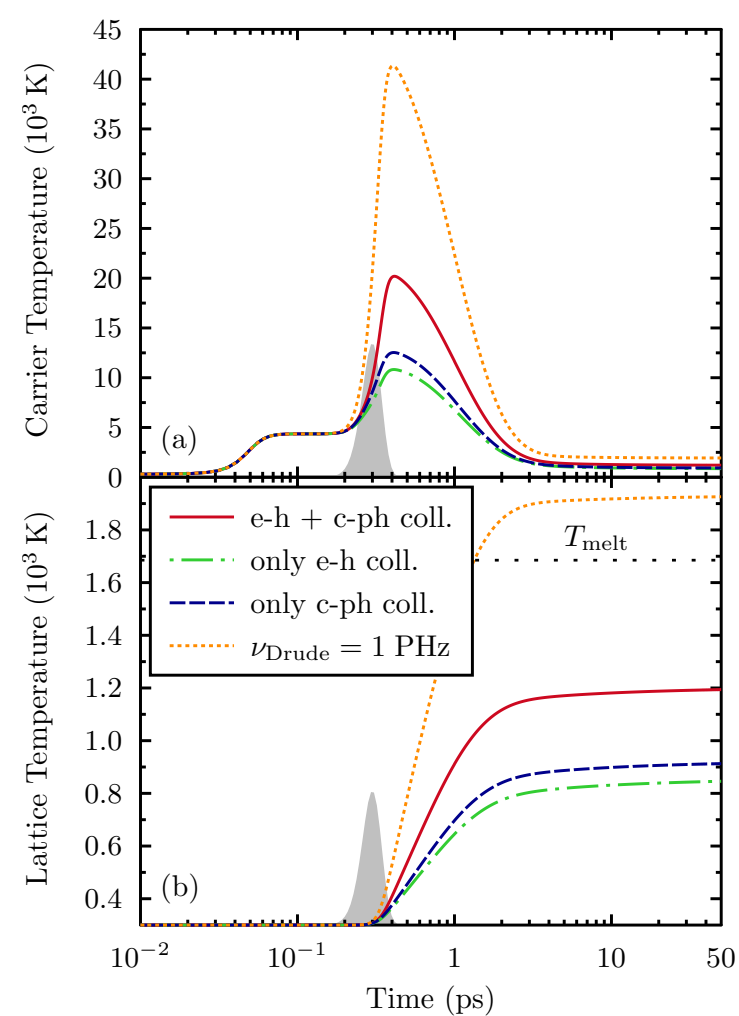

FIG. 6. (Color online) Influence of the carrier collision frequency used in the Drude model on carrier (a) and lattice temperature (b). Carrier density is not shown because there are only minor differences.

We conclude, that the calculated temperatures depend highly on the assumption on carrier collision frequency. Consequently, this assumption might significantly alter the prediction of damage thresholds. Here, we will compare the damage threshold when considering both types of collisions with thresholds obtained for constant collision frequencies.

Figure 7 depicts the damage thresholds calculated with the full model and, hence, a transient carrier collision frequency (solid red diamonds, same curve appears in Fig. 4) as well as with two different constant collision frequencies. The collision frequency of $1 \mathrm{PHz}$ (blue circles) can be found in literature ${ }^{6}$, while the frequency of $283 \mathrm{THz}$ (green triangles) was determined by fitting to an experimental data point by Allenspacher et al. ${ }^{8}$ for a pulse duration in the picosecond range.

For short pulses with durations up to about $300 \mathrm{fs}$, all calculated damage thresholds are in good agreement with the experimental data. For longer pulses, however, the threshold curves for constant carrier collision frequencies flatten while the damage threshold for a transient frequency continues to increase. Because of the flattening in the threshold curve, the calculation using a constant collision frequency of $1 \mathrm{PHz}$ underestimates the damage threshold for picosecond pulses. While calculations using the lower frequency of $283 \mathrm{THz}$ predict fluences that com- 


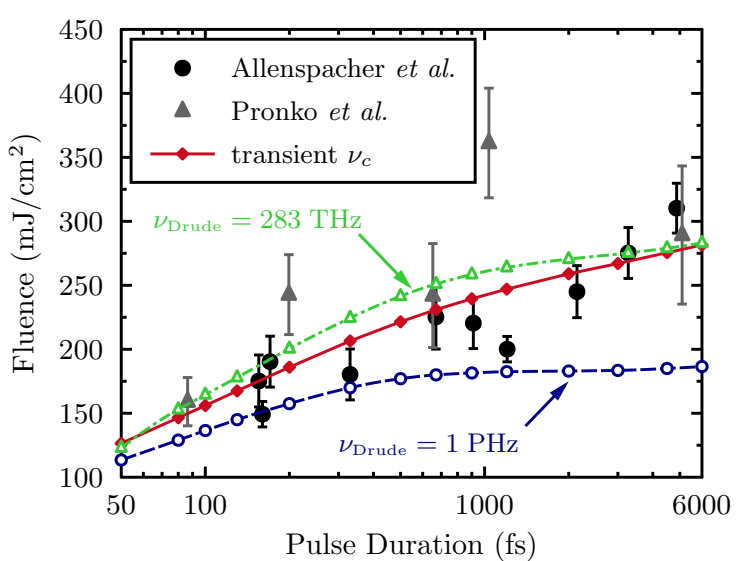

FIG. 7. (Color online) Damage thresholds calculated using the full model with a transient carrier collision frequency as well as two different constant collision frequencies in comparison to experimental data published by Allenspacher et al. ${ }^{8}$ and Pronko et al. ${ }^{72}$

pare very well with experimental results even for picosecond pulses, the further increase in the damage threshold for a transient collision frequency best resembles the experimental data. This increase is due to the fact, that for longer pulses the maximum carrier temperature and thus, according to Eq. (27), the transient electron-hole collision frequency decrease. Consequently, FCA becomes less effective for longer pulse durations which causes the threshold for a transient frequency to increase further.

In summary, we conclude that all thresholds calculated here agree reasonably well with the experimental results for short pulse durations. The threshold calculated using a transient collision frequency is, however, the one that best reproduces the overall behavior indicated by the experimental data. Moreover, no fitting is involved in generating this threshold curve, while it is necessary to fit the constant frequency to achieve an reasonable agreement with the experimental data over a range of pulse durations. Consequently, a transient collision frequency should be applied if a wide range of pulse durations or picosecond pulses are to be treated.

\section{Influence of Degeneracy}

As has been discussed in Sec. II D, the system of equations drastically simplifies when the carrier system is assumed to be non-degenerate (Maxwell-Boltzmann distributed). In the following, we will investigate, whether this assumption is justified in laser-excited silicon. For that purpose, we use a transient carrier collision frequency and again neglect transport.

The distribution functions of electrons and holes enter the equation for carrier energy density (11) and consequently the calculation of carrier temperature. Thus, when abandoning the assumption of a classical non-

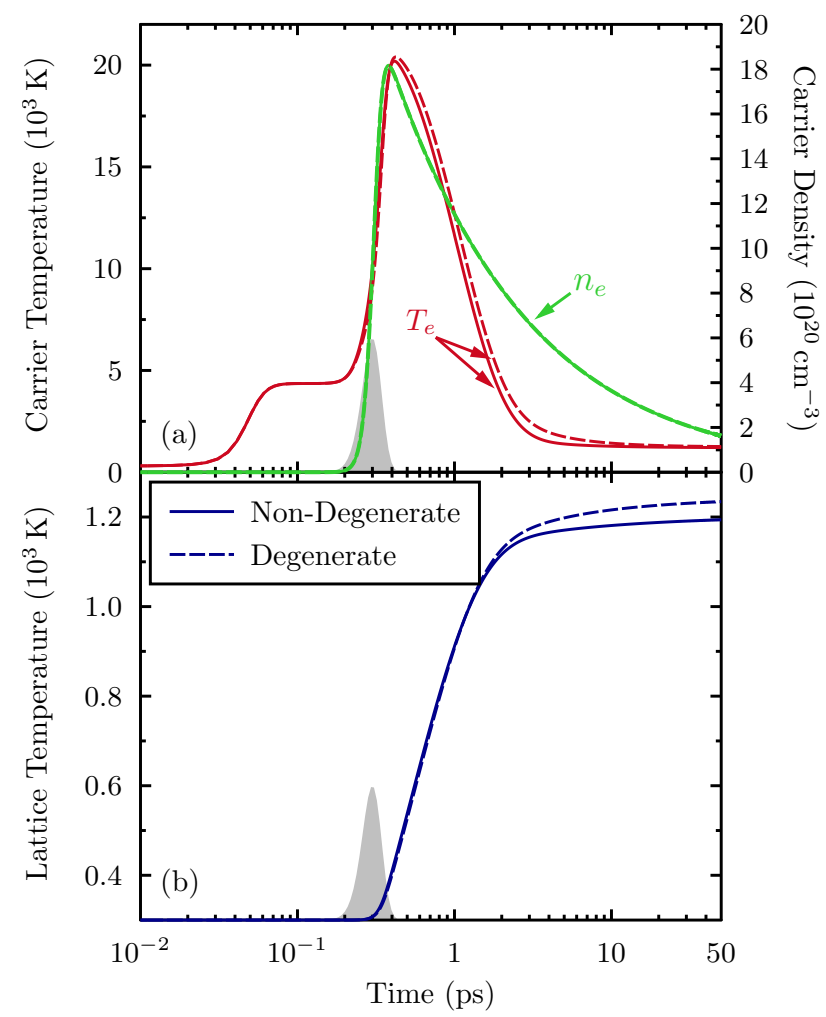

FIG. 8. (Color online) Carrier density and temperature (a) as well as lattice temperature (b) calculated assuming either a Maxwell-Boltzmann distributed or a Fermi distributed carrier system.

degenerate carrier gas, the expression for carrier energy (18) becomes invalid and the full equation (11) has to be used instead. Consequently, the calculation of carrier temperature following Eq. (15) also becomes more laborious.

Figure 8 shows carrier densities and temperatures as well as lattice temperatures calculated assuming a nondegenerate carrier gas and allowing the carrier gas to become degenerate, respectively. While the choice of carrier distribution function has almost no influence on carrier density, there are differences in carrier temperature and, especially, lattice temperature (Fig. 8(b)).

To check whether and when the carrier system becomes degenerate, carrier temperature and Fermi temperatures of electrons and holes (19) are depicted in Fig. 9(a). If the Fermi temperature of electrons and holes is larger than carrier temperature, the respective carrier system is degenerate and should be described by a Fermi distribution. While the electron system is degenerate for a short time during the laser pulse and then again after the laser pulse, the hole system only becomes degenerate after the pulse at later times than the electrons.

Alternatively, we can check whether the chemical potential of the electrons (holes) is positioned inside the conduction (valence) band, which is another indication for degeneracy. It is equivalent to the question whether 


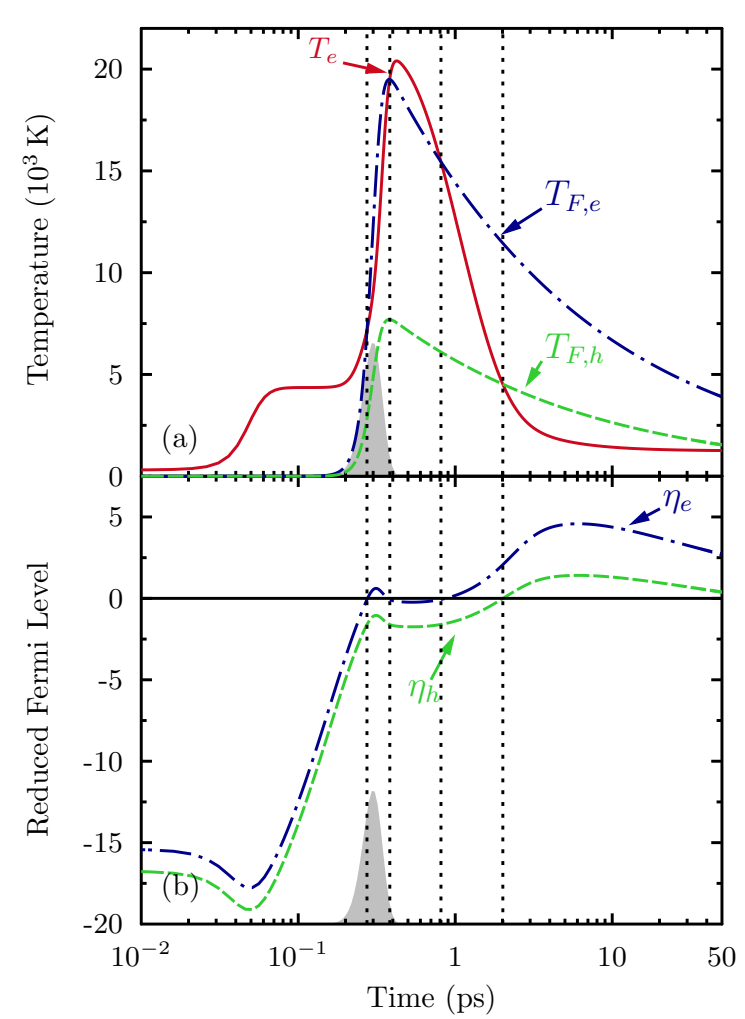

FIG. 9. (Color online) Carrier temperature in comparison with Fermi temperature for electrons and holes, respectively, (a) and reduced Fermi levels of electrons and holes (b).

the reduced Fermi levels of electrons and holes (5) are negative (chemical potential is positioned outside the band) or positive (chemical potential is positioned inside the band). Figure 9(b) shows the reduced Fermi levels of electrons and holes. It is obvious that this criterion indicates a degenerate electron and hole system in the same time intervals as the comparison with Fermi temperature. In addition, we see that the degeneracy of electrons is more pronounced than the degeneracy of holes because the reduced Fermi level of the electrons is larger due to different effective masses. This means in turn that the chemical potential of the electrons is positioned deeper within the conduction band than the one of the holes is in the valence band.

The predicted damage thresholds, shown in Fig. 10, are nearly identical and are both in very good agreement with the experimental data.

We conclude that though the electron as well as the hole system become degenerate during and after irradiation, it suffices to apply a Maxwell-Boltzmann distribution and, thus, to consider a non-degenerate carrier system, when estimating damage thresholds. This immensely reduces computational effort.

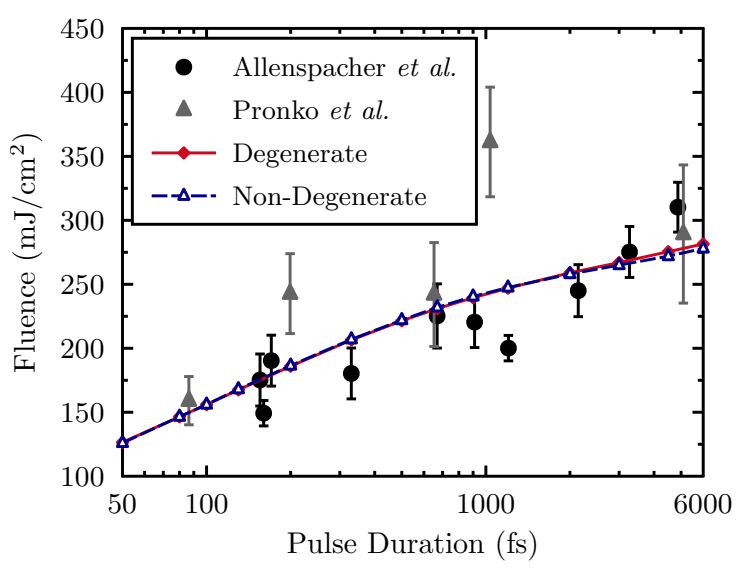

FIG. 10. (Color online) Damage threshold calculated assuming either a Maxwell-Boltzmann distribution or using the full model considering a Fermi distributed carrier system in comparison to experimental data published by Allenspacher et $a l .^{8}$ and Pronko et al. ${ }^{72}$

\section{Influence of Transport}

In this section, we will investigate transport effects. To that end, we consider both particle (7) and energy (9) transport. In all following calculations, the full system of equations is solved, thus allowing for degeneracy of the electron and hole system, respectively.

Figure 11 depicts carrier and lattice temperature as well as carrier density at the surface calculated either considering or neglecting transport. The maxima of carrier and lattice temperature are lower when transport is considered, because energy dissipates away from the surface. In addition, while lattice temperature still increases even on long times scales of tens of picoseconds in the case without transport, it reaches a maximum and starts to decrease on longer timescales when transport is considered.

For carrier density things are a bit different. While the maximum carrier density shows the same behavior as the temperatures, after about 5 ps the density at the surface becomes higher when transport is considered in comparison to the case without transport. This behavior of surface carrier density can be attributed to carrier confinement which has been investigated by Preston and van Driel ${ }^{1,11}$ for silicon irradiated with picosecond and nanosecond pulses.

Let us now briefly have a look at the influence of transport effects on the estimated damage thresholds.

Figure 12 depicts the damage thresholds calculated using the full model (red diamonds, same curve appears in Fig. 4, 7 and 10) and neglecting transport (blue triangles) in comparison with experimental data published by Allenspacher et al. ${ }^{8}$ and Pronko et al..$^{72}$

The damage thresholds estimated neglecting transport are lower than those when considering transport but show the same overall behavior with increasing pulse duration 


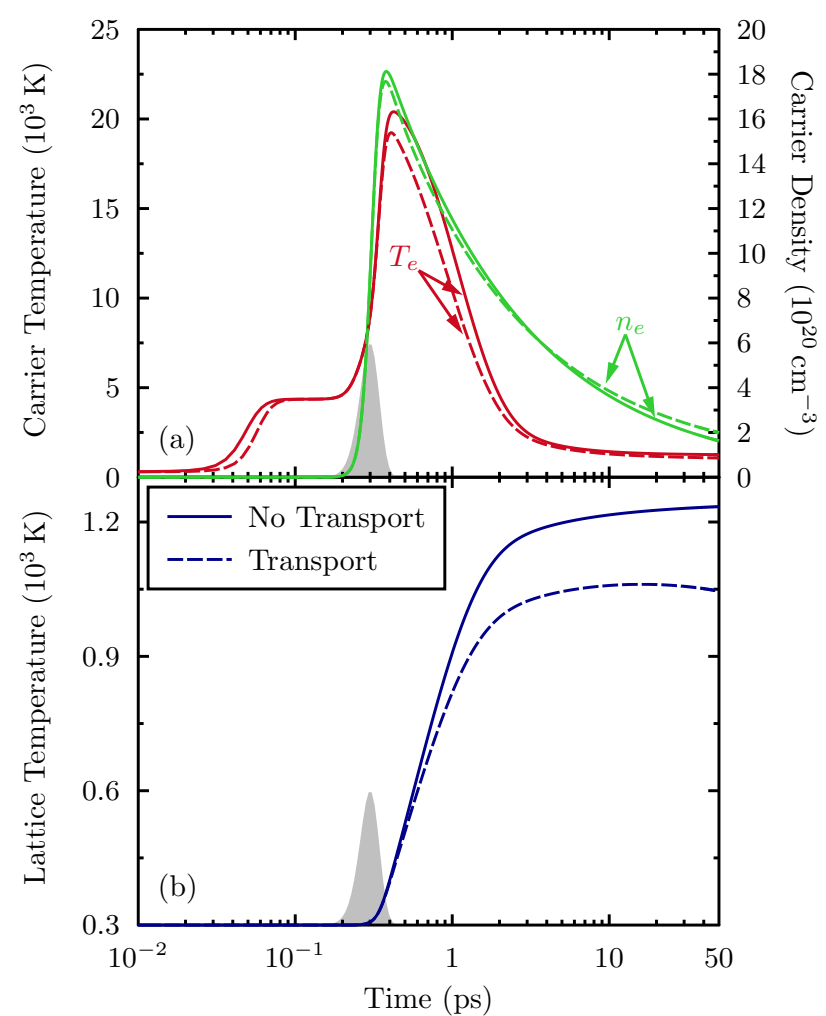

FIG. 11. (Color online) Carrier density and temperature (a) as well as lattice temperature (b) at the surface calculated either considering or neglecting transport.

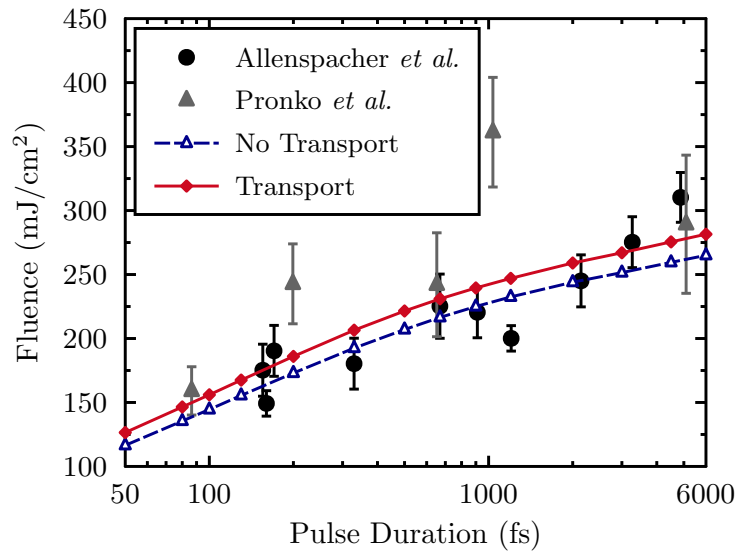

FIG. 12. (Color online) Damage thresholds calculated either considering or neglecting transport in comparison with experimental data published by Allenspacher et al. ${ }^{8}$ and Pronko et $a l .^{72}$

and are still in good agreement with experimental data. The threshold being lower when neglecting transport can be explained by the fact that in this case no heat is carried away from the surface.

Thus, we conclude that as long as only the temperature evolution at the surface is of interest (e.g. for the estimation of damage thresholds) and no detailed investigation of temperature or density profiles is necessary, it is sufficient to implement an nTTM neglecting transport, which considerably reduces computational efforts.

\section{SUMMARY AND CONCLUSION}

In this work, we extended the nTTM first presented by van Driel $^{11}$ to account for the changes in optical parameters, namely reflectivity and FCA coefficient, due to the highly transient free carrier density during the excitation with femtosecond laser pulses.

For the irradiation of silicon with a 100 fs-laser pulse at $800 \mathrm{~nm}$, we analyzed the influence of the transient optical properties. We conclude, that it is of utter importance to consider changes in reflectivity and FCA coefficient due to the changing carrier density. This can not only be seen in the reflectivity, temperature and density evolution but is also clearly reflected in the calculated damage thresholds. Comparison with experimental data shows that the $T$-expression often used with the nTTM in earlier works strongly overestimates the threshold. Damage thresholds calculated with our improved approach using a Drude model considering both electron-hole and carrierphonon collisions are, however, in very good agreement with experimental data even over a wide range of pulse durations. Note that we did not fit any parameter of our model to reproduce experimental damage thresholds.

Moreover, we found that the choice of carrier collision frequency used in the Drude model strongly influences the lattice temperature and, consequently, the calculated damage threshold. Thus, we conclude that it does not suffice to assume a constant carrier collision frequency at least when treating a wide range of pulse durations. In this case, a transient carrier collision frequency considering both, electron-hole collisions and carrier-phonon collisions, should be applied to best reproduce experimental data.

We analyzed the influence of the distribution function assumed for the carriers and found that while both electron and hole system become degenerate when excited with the laser pulse, it suffices to assume a MaxwellBoltzmann distribution for the carriers when estimating damage thresholds.

Furthermore, we investigated transport of carriers and energy and found that the influence of the transient band gap during the excitation can lead to carrier confinement in a region below the incident surface. Moreover, we found that damage thresholds calculated neglecting transport are a bit lower than those when considering transport but show the same overall behavior with increasing pulse duration and are still in good agreement with experimental data.

We therefore conclude, that it suffices to use an nTTM neglecting transport and degeneracy effects. This immensely reduces computational effort in comparison to a full calculation. 
Finally, we conclude that our approach yields highly satisfying results in agreement with experimental data once a Drude model considering a transient carrier collision frequency is implemented. The improved nTTM considering transient optical parameters therefore is a powerful tool to describe laser-excited semiconductors.

\section{ACKNOWLEDGMENTS}

The authors thank K. Sokolowski-Tinten and V. P. Lipp for helpful suggestions. Financial support by the Deutsche Forschungsgemeinschaft through the Emmy Noether (grant no. RE 1141/11) and the Heisenberg program (grant no. RE 1141/15) is gratefully acknowledged.

${ }^{1}$ J. S. Preston and H. M. van Driel, Phys. Rev. B 30, 1950 (1984). ${ }^{2}$ X. Y. Wang, D. M. Riffe, Y.-S. Lee, and M. C. Downer, Phys. Rev. B 50, 8016 (1994).

${ }^{3}$ J. R. Goldman and J. A. Prybyla, Phys. Rev. Lett. 72, 1364 (1994).

${ }^{4}$ B. N. Chichkov, C. Momma, S. Nolte, F. Alvensleben, and A. Tünnermann, Applied Physics A 63, 109 (1996).

${ }^{5}$ T. Sjodin, H. Petek, and H.-L. Dai, Phys. Rev. Lett. 81, 5664 (1998).

${ }^{6}$ K. Sokolowski-Tinten and D. von der Linde, Phys. Rev. B 61, 2643 (2000).

${ }^{7}$ A. J. Sabbah and D. M. Riffe, Phys. Rev. B 66, 165217 (2002).

${ }^{8}$ P. Allenspacher, B. Hüttner, and W. Riede, Proc. SPIE 4932, 358 (2003).

${ }^{9}$ L. Englert, M. Wollenhaupt, L. Haag, C. Sarpe-Tudoran, B. Rethfeld, and T. Baumert, Applied Physics A 92, 749 (2008).

${ }^{10}$ S. I. Anisimov, B. L. Kapeliovich, and T. L. Perel'man, Zh. Eksp. Teor. Fiz. 66, 776 (1974), [Sov. Phys. JETP 39, 375 (1974)].

${ }^{11}$ H. M. van Driel, Phys. Rev. B 35, 8166 (1987).

${ }^{12}$ B. Rethfeld, A. Kaiser, M. Vicanek, and G. Simon, Applied Physics A 69, S109 (1999).

${ }^{13}$ B. Rethfeld, Phys. Rev. Lett. 92, 187401 (2004).

${ }^{14}$ N. M. Bulgakova, R. Stoian, A. Rosenfeld, I. V. Hertel, and E. E. B. Campbell, Phys. Rev. B 69, 054102 (2004).

${ }^{15}$ N. M. Bulgakova, R. Stoian, A. Rosenfeld, I. V. Hertel, W. Marine, and E. E. B. Campbell, Applied Physics A 81, 345 (2005).

${ }^{16}$ B. H. Christensen and P. Balling, Phys. Rev. B 79, 155424 (2009).

${ }^{17}$ D. Autrique, G. Clair, D. L'Hermite, V. Alexiades, A. Bogaerts, and B. Rethfeld, Journal of Applied Physics 114, 023301 (2013).

${ }^{18} \mathrm{~A}$. Vogel and V. Venugopalan, Chemical Reviews 103, 577 (2003).

${ }^{19}$ M. Li, K. Mori, M. Ishizuka, X. Liu, Y. Sugimoto, N. Ikeda, and K. Asakawa, Applied Physics Letters 83, 216 (2003).

${ }^{20}$ A. Rodriguez, M. Echeverría, M. Ellman, N. Perez, Y. K. Verevkin, C. S. Peng, T. Berthou, Z. Wang, I. Ayerdi, J. Savall, and S. M. Olaizola, Microelectronic Engineering 86, 937 (2009).

${ }^{21}$ D. Bäuerle, Laser Processing and Chemistry, 4th ed. (Springer, Berlin, 2011).

${ }^{22}$ B. Rethfeld, K. Sokolowski-Tinten, D. von der Linde, and S. I. Anisimov, Applied Physics A 79, 767 (2004).

${ }^{23}$ D. Bejan and G. Raşeev, Phys. Rev. B 55, 4250 (1997).

${ }^{24}$ A. Kaiser, B. Rethfeld, M. Vicanek, and G. Simon, Phys. Rev. B 61, 11437 (2000).

${ }^{25}$ L. Pietanza, G. Colonna, S. Longo, and M. Capitelli, Applied Physics A 79, 1047 (2004).

${ }^{26}$ L. D. Pietanza, G. Colonna, S. Longo, and M. Capitelli, The European Physical Journal D 45, 369 (2007).

${ }^{27}$ B. Y. Mueller and B. Rethfeld, Phys. Rev. B 87, 035139 (2013).

${ }^{28}$ N. S. Shcheblanov and T. E. Itina, Applied Physics A 110, 579 (2013).
${ }^{29}$ F. Gao, L. W. Campbell, R. Devanathan, X. L. Xie, Y. Zhang, A. J. Peurrung, and W. J. Weber, Nuclear Instruments and Methods in Physics Research Section B: Beam Interactions with Materials and Atoms 255, 286 (2007).

${ }^{30}$ F. Gao, L. W. Campbell, R. Devanathan, Y. Xie, L. R. Corrales, A. J. Peurrung, and W. J. Weber, Nuclear Instruments and Methods in Physics Research Section A: Accelerators, Spectrometers, Detectors and Associated Equipment 579, 292 (2007).

${ }^{31}$ N. Medvedev, U. Zastrau, E. Förster, D. O. Gericke, and B. Rethfeld, Phys. Rev. Lett. 107, 165003 (2011).

${ }^{32}$ V. Laporta, L. D. Pietanza, and G. Colonna, Nuclear Instruments and Methods in Physics Research Section A: Accelerators, Spectrometers, Detectors and Associated Equipment 636, 67 (2011).

${ }^{33}$ A. D. Bristow, N. Rotenberg, and H. M. van Driel, Applied Physics Letters 90, 191104 (2007).

${ }^{34}$ Y. P. Varshni, Physica 34, 149 (1967).

${ }^{35}$ G. E. Jellison and F. A. Modine, Phys. Rev. B 27, 7466 (1983).

${ }^{36}$ J. K. Chen, D. Y. Tzou, and J. E. Beraun, International Journal of Heat and Mass Transfer 48, 501 (2005).

${ }^{37}$ Y. Gan and J. K. Chen, Computer Physics Communications 183, 278 (2012).

${ }^{38}$ B. Rethfeld, A. Rämer, N. Brouwer, N. Medvedev, and O. Osmani, Nuclear Instruments and Methods in Physics Research Section B: Beam Interactions with Materials and Atoms 327, 78 (2014).

${ }^{39}$ M. Galassi, J. Davies, J. Theiler, B. Gough, G. Jungman, P. Alken, M. Booth, and F. Rossi, GNU Scientific Library Reference Manual, 3rd ed. (Network Theory Ltd, 2009).

${ }^{40}$ D. P. Korfiatis, K.-A. T. Thoma, and J. C. Vardaxoglou, Journal of Physics D: Applied Physics 40, 6803 (2007).

${ }^{41}$ M. I. Gallant and H. M. van Driel, Phys. Rev. B 26, 2133 (1982).

${ }^{42}$ J. P. Callan, A. T.-M. Kim, L. Huang, and E. Mazur, Chemical Physics 251, 167 (2000).

${ }^{43}$ W. G. Spitzer and H. Y. Fan, Phys. Rev. 106, 882 (1957).

${ }^{44}$ M. Fox, Optical Properties of Solids, 2nd ed. (Oxford University Press, Oxford, 2010).

${ }^{45} \mathrm{P}$. Balling and J. Schou, Reports on progress in physics 76, 036502 (2013).

${ }^{46}$ F. Quéré, S. Guizard, and P. Martin, EPL (Europhysics Letters) 56, 138 (2001).

${ }^{47}$ B. Rethfeld, H. Krutsch, and D. Hoffmann, Contributions to Plasma Physics 50, 16 (2010).

${ }^{48}$ E. P. Silaeva, N. S. Shcheblanov, T. E. Itina, A. Vella, J. Houard, N. Sévelin-Radiguet, F. Vurpillot, and B. Deconihout, Applied Physics A 110, 703 (2013).

${ }^{49}$ T. Y. Choi and C. P. Grigoropoulos, Journal of Applied Physics 92, 4918 (2002).

${ }^{50}$ H. Zhang, D. van Oosten, D. M. Krol, and J. I. Dijkhuis, Applied Physics Letters 99, 231108 (2011).

${ }^{51}$ Y. Gan and J. K. Chen, Applied Physics A: Materials Science \& Processing 105, 427 (2011).

${ }^{52}$ B. Rethfeld, O. Brenk, N. Medvedev, H. Krutsch, and D. H. H. Hoffmann, Applied Physics A 101, 19 (2010).

${ }^{53}$ N. Medvedev, B. Ziaja, M. Cammarata, M. Harmand, and S. Toleikis, Contributions to Plasma Physics 53, 347 (2013).

${ }^{54}$ K. Wædegaard, M. Frislev, and P. Balling, Applied Physics A 110, 601 (2013).

${ }^{55}$ D. Hulin, M. Combescot, J. Bok, A. Migus, J. Y. Vinet, and A. Antonetti, Phys. Rev. Lett. 52, 1998 (1984).

${ }^{56}$ M. Combescot and R. Combescot, Phys. Rev. B 35, 7986 (1987).

${ }^{57}$ B. E. Sernelius, Phys. Rev. B 39, 10825 (1989).

${ }^{58}$ B. H. Christensen, K. Vestentoft, and P. Balling, Applied Surface Science 253, 6347 (2007).

${ }^{59}$ N. Medvedev and B. Rethfeld, Journal of Applied Physics 108, 103112 (2010).

${ }^{60}$ N. Del Fatti, C. Voisin, M. Achermann, S. Tzortzakis, D. Christofilos, and F. Vallée, Phys. Rev. B 61, 16956 (2000).

${ }^{61}$ R. Binder, H. S. Köhler, M. Bonitz, and N. Kwong, Phys. Rev. B 55, 5110 (1997). 
${ }^{62}$ W. H. Press, S. A. Teukolsky, W. T. Vetterling, and B. P. Flannery, Numerical Recipes, 3rd ed. (Cambridge University Press, Cambridge, 2007).

${ }^{63}$ J. Hohlfeld, S.-S. Wellershoff, J. Güdde, U. Conrad, V. Jähnke, and E. Matthias, Chemical Physics 251, 237 (2000).

${ }^{64}$ M. Bonn, D. N. Denzler, S. Funk, M. Wolf, S.-S. Wellershoff, and J. Hohlfeld, Phys. Rev. B 61, 1101 (2000).

${ }^{65}$ K. Vestentoft and P. Balling, Applied Physics A 84, 207 (2006).

${ }^{66}$ J. Byskov-Nielsen, J.-M. Savolainen, M. S. Christensen, and
P. Balling, Applied Physics A 103, 447 (2011).

${ }^{67}$ H. Barber, Solid-State Electronics 10, 1039 (1967).

${ }^{68}$ R. F. Wood and G. E. Giles, Phys. Rev. B 23, 2923 (1981).

${ }^{69}$ D. Agassi, Journal of Applied Physics 55, 4376 (1984).

70 J. Dziewior and W. Schmid, Applied Physics Letters 31, 346 (1977).

${ }^{71}$ E. D. Palik, Handbook of Optical Constants of Solids (Academic Press, San Diego, 1985)

${ }^{72}$ P. P. Pronko, P. A. VanRompay, C. Horvath, F. Loesel, T. Juhasz, X. Liu, and G. Mourou, Phys. Rev. B 58, 2387 (1998). 